\title{
Splenic infarction and spontaneous rupture in a patient with paratyphoid
}

\author{
D P Wickramasinghe ${ }^{1}$, D N Samarasekera ${ }^{1}$
}

Ceylon Medical Journal 2012; 57: 130-131

\section{Introduction}

Paratyphoid is caused by Salmonella enterica serotype paratyphi A, B or C. There are only subtle clinical differences between the typhoid and paratyphoid but there is no difference between the response to therapy or frequency of complications [1]. Splenic infarction is an identified complication of typhoid and paratyphoid $[2,3]$. However, there are only a few reports of spontaneous splenic rupture due to typhoid but hardly any due to paratyphoid $[4,5]$. We describe a patient with paratyphoid A, who developed splenic infarction and subsequent rupture.

\section{Case report}

A 76-year old man with a history of diabetes mellitus presented with obstructive jaundice. He had developed jaundice 2 weeks prior to admission with subsequent pale stools and dark urine. He also complained of colicky pain in the right hypochondrium (RHC) and episodic high grade fever with chills and rigors. On admission, he was febrile and deeply icteric. There was RHC tenderness and hepatomegaly but no palpable splenomegaly.

The first ultrasound scan had shown increased coarse pattern suggestive of fatty liver and multiple gallstones in the gall bladder (GB) with a thickened wall but no dilatation of the common bile duct (CBD). There was mild splenomegaly of $12 \mathrm{~cm}$. The ultrasound done on day 2 following admission identified all the above and a mild dilatation of the CBD (diameter $1.1 \mathrm{~cm}$ ). However, neither an obstructing CBD calculus nor any focal abnormalities of the spleen were identified.

There was a persistent leukocytosis (WBC - 21,500 / $\mathrm{mm}^{3}$ ) with a neutrophilia $(94.5 \%)$ and a relative lymphopenia (3.7\%). Liver enzymes and alkaline phosphatase levels were elevated (ALT - $56 \mathrm{u} / 1$, AST- 33 $\mathrm{u} / \mathrm{l}$, ALP- $912 \mathrm{u} / \mathrm{l}$ ). Total bilirubin and direct fraction were also elevated ( $563 \mu \mathrm{mol} / 1$ and $209 \mu \mathrm{mol} / 1$ respectively). Therefore, a diagnosis of acute cholecystitis was made and the patient was started on intravenous ciprofloxacin. However, the preliminary blood culture report at 24 hours failed to identify any growth.

He developed spiking fever with an irregularly irregular pulse and rising total and direct bilirubin. ACT of the abdomen showed infarction of the spleen with gas inside the splenic parenchyma and free gas under the diaphragm.

Given the progressive deterioration, free gas in the peritoneum and the suppuration in the spleen, he underwent an exploratory laparotomy. Intraoperatively, the spleen was found to have ruptured with purulent blood stained fluid collection in the left paracolic gutter. The remaining necrotic tissue of the spleen was excised and a cholecystectomy was performed. All specimens were sent for microbiological assessment. He was treated in the intensive care unit following surgery but on the 5 th post operative day developed a respiratory arrest. Resuscitation was unsuccessful.

The blood culture had a growth of Salmonella enterica serotype paratyphi A at the end of 7 days. No organisms were isolated from the specimens taken at surgery. Histology showed infarction of splenic tissue with extensive areas of necrosis mainly involving the red pulp and sparse neutrophils in the parenchyma.

\section{Discussion}

Though the patient's initial presentation favoured a suspicion of CBD obstruction by gall stones, retrospectively at least, the 2 conditions seem to be coincidental at most. The relative lymphopenia and the non-dilated CBD discovered at surgery were in favour of the diagnosis of typhoid. The hepatomegaly, jaundice and biochemical derangement were suggestive of typhoid hepatitis and the mild elevation of transaminases was further in favour of this $[5,6]$. Hepatomegaly is a consistent finding in all cases of jaundice in patients with typhoid [6]. The atrial fibrillation (AF) may have been a paroxysmal episode which was precipitated by hypovolaemia or due to typhoid myocarditis.

Splenic rupture has been described in typhoid but very rarely in paratyphoid $[4,7]$. Available evidence indicates a high mortality $(50 \%)$ in patients older than 40 years, pre-existing splenomegaly and total splenectomy is the primary procedure for spontaneous splenic rupture [4]. Diabetes mellitus has been identified as a predisposing factor of typhoid splenic abscess [8].

Therefore it is important to consider the possibility of enteric fever in a patient with features of obstructive jaundice and cholangitis not responding to standard

${ }^{1}$ Department of Surgery, Faculty of Medicine, University of Colombo, Sri Lanka.

Correspondence: DNS, e-mail: <samarasekera58@yahoo.co.uk>. Received 4 August 2011 and revised version accepted 18 May 2012. Competing interests: none declared. 
antibiotics, especially in the tropics (i.e. as typhoid fever is endemic) as the consequence of delayed detection can be fatal.

\section{References}

1. Abdel Wahab MF, Haseeb AN, Hamdy HS, Awadalla YA. Comparative study between paratyphoid A and typhoid fever cases. Journal of the Egyptian Public Health Association 1996; 71: 539-51.

2. Mehta LK, Arya SC and Mathai G. Infarction of spleen in typhoid fever. Saudi Medical Journal 2007; 28: 271-2.

3. Gautier-Benoit C, Pontac MO, Hochedez G, Houcke M. [Splenic infarct revealing paratyphoid fever in a child]. Journal de la Faculte de Medecine et de Pharmacie de l'Universite de Lille 1975: 20: 630-1.
4. Renzulli P, Hostettler A, Schoepfer AM, Gloor B, Candinas D. Systematic review of atraumatic splenic rupture. British Journal of Surgery 2009; 96: 1114-21.

5. Khosla SN. Typhoid hepatitis. Postgraduate Medical Journal 1990; 66: 923-5.

6. Ramachandran S, Godfrey JJ, Perera MV. Typhoid hepatitis. Journal of American Medical Association 1974; 230: $236-40$.

7. Jezyna C, Markiewicz W. [An unusual case of spontaneous splenic rupture in paratyphoid B]. Wiadomosci lekarskie 1987; 40: 1332-5.

8. Torres JR, Gotuzzo E, Isturiz R, et al. Salmonellal splenic abscess in the antibiotic era: a Latin American perspective. Clinical Infectious Diseases 1994; 19: 871-5. 